\title{
META ANÁLISIS SOBRE LA APLICACIÓN DEL MODELO DE SOLUCIÓN DE PROBLEMA EN LA LEGISLACIÓN DE COMUNIDADES ESPECIALES ${ }^{~}$
}

\section{Rubén Estremera Jiménez ${ }^{2}$}

\section{Introducción:}

Este trabajo tiene el propósito de analizar críticamente el modelo de solución de problemas utilizado en un escrito en la cual se analiza la ley de Comunidades Especiales (CE) a partir del modelo positivista de solución de problemas (Estremera, 2002). Se repiensa el análisis de este modelo a partir de algunos de sus supuestos positivistas y de sus implicaciones epistemológicas. En dicho trabajo se da por sentado que el modelo clásico de solución de problemas es el más adecuado, sin cuestionar sus implicaciones o contradicciones en el escenario de trabajo social. Hay que destacar que el modelo de solución de problema se utiliza en trabajo social con individuo, grupo, comunidad y análisis de política pública. Hay cierta naturalidad en la retórica cotidiana al aceptar este método como único y permanente, obviando lo cambiante de la realidad y posibles conflictos con los valores de equidad y justicia de la profesión de trabajo social. ${ }^{3}$

Así, el interés que orienta este análisis es la incertidumbre aguda de haber analizado la política pública de Comunidades Especiales desde una perspectiva instrumental, positivista, no haber problematizado en torno a su complejidad y sus implicaciones intelectuales de tal proceder. Por lo tanto, hay un interés crítico de refutar lo hecho a partir de otros conceptos epistemológicos. Se orienta el análisis con criterios interpretativos que trascienden el análisis de política pública

\footnotetext{
${ }^{1}$ Artículo basado en la ponencia presentada en el V Encuentro de Política Social y Trabajo Social, celebrado en la Universidad de Costa Rica, 27-29 de mayo de 2003.

${ }^{2}$ Catedrático Asociado del Departamento de Trabajo Social de la Universidad de Puerto Rico. ${ }^{3}$ Durkheim (1978) en su escrito las reglas del método sociológico cuestionó el uso de un método permanente debido a lo cambiante de la realidad estudiada. Por consiguiente, es cuestionable proponer que existe un método único de estudiar un fenómeno y no una diversidad de acuerdo a la realidad.
} 
propuesto por el modelo de solución de problema (Gilbert y Jones, 1984).

Surgen interrogantes que requieren contestación para poder problematizar en torno al tipo de estrategia a utilizar en la política social vigente para lidiar con el problema de la pobreza en Puerto Rico. Por ejemplo: ¿Es posible analizar una política social sin desnaturalizar los conceptos principales de la legislación? ¿La pobreza es un problema o un fenómeno social de contradicciones?, ¿Por qué no utilizar otros modelos de análisis orientado hacia las contradicciones?, ¿Qué métodos de análisis no son adecuados para estudiar la legislación de comunidades especiales? Estas y otras interrogantes surgen conjeturalmente y las mismas no tienen contestación en el momento. No obstante, es el interés de este escrito aclarar algunas de estas interrogantes de tal manera que al final de la argumentación se establezca cuál debe ser el problema de estudio de las conjeturas aquí expuestas.

Primeramente, se analiza brevemente la política pública del Estado Libre Asociado de Puerto Rico en torno a la ley \#1 de Comunidades Especiales (CE) sometida por la Hon. Sila María Calderón, Gobernadora de Puerto Rico, el 1 de marzo de 2001. Luego, se describe el modelo de análisis de solución de problemas utilizado para estudiar la legislación de CE. Se analiza críticamente las posturas epistemológicas de la cual parte este modelo. Se evalúan los supuestos epistemológicos del escrito objeto de análisis, dando atención particular a lo metodológico y a la relación teoría-praxis. Finalmente, se reformulan los problemas del análisis basado en la argumentación crítica.

\section{Metas y objetivos de la política existente}

El 1 de marzo de 2001 la Hon. Sila M. Calderón, Gobernadora de Puerto Rico firmó la ley \#1 de Comunidades Especiales dirigida al:

"Desarrollo Integral de las Comunidades Especiales de Puerto Rico, a fin de establecer la política pública del Estado Libre Asociado de Puerto Rico en lo relativo al desarrollo integral de las comunidades especiales del país; establecer guías a considerar para identificar a las comunidades especiales; crear 
la Oficina del Coordinador General para el Financiamiento Socio-Económico y la Autogestión, el cargo de Coordinador General, el Fondo para el Desarrollo Socio-Económico de las Comunidades Especiales y el Consejo para las Comunidades Especiales; y para asignar recursos para la organización de la oficina y el inicio del programa" (p.1).

En la exposición de motivo no se hace explícito la meta que orienta la ley, por lo que se procede a inferir la misma: Mejorar las condiciones de vida de personas de bajos ingresos mediante la organización comunitaria y la autogestión en su desarrollo socioeconómico. La política pública se fundamenta en el principio de la autogestión y apoderamiento comunitario. Este principio conlleva la toma de conciencia de los miembros de la comunidad para tomar el control de sus problemas y orientarse hacia su solución basada en el esfuerzo propio.

La ley establece procedimientos generales para determinar que constituye una comunidad especial. Los criterios para identificar una comunidad especial son: bajos niveles socioeconómicos, problemas ambientales, deficiencia en la infraestructura de las comunidades, deficiencias en la provisión de servicios del gobierno y viviendas inadecuadas. A continuación se presentan algunos acontecimientos históricos que relacionan la profesión de trabajo social con política social y su relación con el modelo de solución de problemas.

\section{Problematización}

\section{Historia: Trabajo Social y Análisis de Política Pública}

La política social es un área de intervención profesional que se hizo parte del saber profesional y académico en el campo del trabajo social puertorriqueño en la década del 70 al 80 (Guardiola, 2000). La gran influencia del trabajo social clínico iniciado en la década del 30 y la inserción del trabajador social en puestos de agencias del gobierno fueron factores principales para que la orientación de las prácticas del trabajo social se desligaran de proyectos comunitarios, de acción y política social. La profesión de trabajo social se naturalizó por una perspectiva asistencialista que se caracterizó por proveer servicios en agencias del gobierno (Guardiola, 1998). Se partía del supuesto 
que a los clientes se les ofrecían servicios de educación, salud, social y otros debido a su incapacidad personal para resolver sus problemas. Los problemas de los individuos eran el resultado de su incapacidad personal y no del entrejuego de fuerzas estructurales o ideológicas que condicionaban su quehacer. Se pensaba que la "naturaleza" de la intervención profesional se centraba en trabajar con incapacidades de los individuos, grupos o comunidad y la profesión no se asociaba con el trabajo cultural, político o económico que trasciende lo micro.

La década del 70 al 80 se caracterizó por un cuestionamiento de la naturaleza asistencial del trabajo social. Se retomó la bandera de la acción social como estrategia de intervención de transformación social. La acción social a través de la intervención en la política pública se hizo parte del discurso del trabajador social como arma para lidiar con la injusticia y la equidad.

La profesión de trabajo social fue influenciada notablemente por las corrientes positivistas al proponer pautas de intervención de orientación tecnológica en todas las áreas de trabajo social y en particular a la manera de analizar políticas sociales (Ruiz, 1997). La profesionalización a través de proceso tecno-científico llevó a la profesión a identificarse con métodos de acción social con una orientación objetiva y neutral. Se partía del supuesto que la ciencia estaba desconectada de la ideología. Por consiguiente, un analista de política social se enfoca en los procesos técnicos y no en las ideologías que la orientan. El enfoque tecnológico en la profesión en trabajo social marcó una nueva dirección de cómo interpretar los problemas sociales y nuevas formas científicas para intervenir con los mismos. El modelo de análisis de política pública cónsono con dicha ideología fue el de solución de problemas. Este modelo de orientación racionalista es el que se ha estado utilizando mayormente en algunos cursos de bachillerato, maestría y doctorado en trabajo social de la Universidad de Puerto Rico para interpretar la política pública del país. ${ }^{4}$ En la próxima sección se analiza el significado del modelo de

\footnotetext{
${ }^{4}$ El currículo de los programas de bachillerato, maestría y doctoral tiene unos cursos cuyo objeto de estudio es la política social. En el curso "Política y Servicio del Bienestar Social" (Bachillerato) se utiliza el modelo de solución de problema como método de análisis y en "Teorías para el análisis de política social y el comportamiento organizacional" para analizar las políticas sociales.
} 
solución de problemas y sus implicaciones en la intervención profesional.

\section{Análisis de Política Pública a partir del Modelo de Solución de Problemas}

El análisis de política pública a partir del modelo de solución de problemas parte del supuesto que el proceso para establecer política pública requiere de la aplicación de un sistema instrumental que sigue unos pasos sistemáticos para resolver un problema social. Este modelo se enfoca en conocer como se toman decisiones, como es el funcionamiento del establecimiento de política pública y como debe hacerse política pública (Dye, 1998).

Lo fundamental de este enfoque es que va dirigido a estudiar un proceso para establecer política pública, independientemente de su contenido. Lo importante es conocer el proceso o la secuencia lógica que utilizan para hacer política pública. No se asigna mucha importancia a evaluar con detenimiento la adecuacidad de los contenidos y si la manera en que estos son procesados y organizados. Se considera que si el proceso es sistemático y organizado es bien probable que el resultado también así lo sea.

El modelo sugiere unos pasos "universales" a seguir para agrupar actividades que lo lleven a tomar decisiones: identificar problema, establecer plan para tomar decisión, formular propuesta de política pública, legitimizar política pública, implementar política pública y evaluar política pública. Hay una diversidad de variantes del uso del modelo de solución de problemas en el escenario de análisis de política pública (Dye, 1998, Jones, 1984, Gilbert y Specht, 1984). ${ }^{6}$

\footnotetext{
${ }^{5}$ El modelo de solución de problemas ha sido utilizado, con diversas variantes, como método genérico en distintos escenarios del trabajo social puertorriqueño como la intervención profesional, análisis de política social y procesos administrativos

${ }^{6}$ Estos autores presentan una variedad de modelos racionalistas que enfocan el análisis de la política pública desde diversas perspectivas. Lo común en todos estos modelos es que siguen un proceso secuencial y sistemático y la diferencia es que unos hacen análisishistóricos, políticos y sociales, mientras otros enfocan en análisis estadísticos y económicos.
} 
El modelo de análisis de política pública utilizado en el estudio de la legislación de comunidades Especiales fue el propuesto Gilbert y Specht (1984). El método de este modelo consiste de los siguientes pasos: definir el problema, criterios para seleccionar alternativas, considerar soluciones, alternativas y adopción de soluciones, establecer tácticas y tareas, establecer plan de implantación, establecer sistemas de acción y recursos, evaluar si se resolvió el problema, tomar decisiones si se resolvió o no el problema. Este escrito analizará críticamente el método de análisis de solución de problemas utilizado en dicho ensayo con otros lentes epistemológicos que lleven a nuevas interpretaciones e intervenciones.

\section{Análisis crítico}

\section{Instrumental}

El modelo de solución de problemas utilizado para analizar la política pública de las Comunidades Especiales responde a supuestos epistemológicos positivizantes que se manifiestan a través del método de análisis y la manera de concebir la relación teoría práctica. Aunque hay otros supuestos epistemológicos que pueden ser considerados en el análisis y evaluación del modelo de solución de problemas ${ }^{7}$, nos limitaremos a analizar críticamente estos dos supuestos para evidenciar la orientación epistemológica de la cual parten. Además, estos supuestos epistemológicos se contraponen con otros supuestos epistemológicos popperianos que lleven a interpretar la realidad objeto de estudio desde otra perspectiva.

Una característica positivizante del modelo de solución de problema utilizado para analizar y evaluar la legislación de Comunidades Especiales es su énfasis en lo instrumental. El énfasis en lo instrumental se manifiesta en la importancia asignada al proceso de analizar una política pública. En el análisis de la legislación de las Comunidades Especiales se le asignó mayor importancia al proceso

\footnotetext{
${ }^{7} \mathrm{El}$ modelo de solución de problemas se utiliza como parte del enfoque generalista del currículo del Departamento de Trabajo Social y se aplica a familia, grupo, comunidad y política social.
} 
(seguir secuencias y pasos en forma sistemática) de análisis que a los contenidos de la legislación. La formalización del método de análisis a través de unos pasos restó importancia al estudio e interpretación del problema objeto de estudio. Se asignó gran esfuerzo en aplicar un procedimiento de estudio riguroso y detallado (analítico) para comprender el proceso legislativo y no su sustantividad. No se analizaron el significado de los conceptos de pobreza o autogestión comunitaria desde sus bases epistemológicas o ético político. Esta limitación conceptual dificulta comprender la complejidad de la pobreza y sugerir estrategias para lidiar con la misma.

Otro aspecto cuestionable en el uso del modelo de solución de problemas como método de análisis único para estudiar la política pública en los cursos de bachillerato, maestría y doctorado. El limitar el análisis de política pública a este modelo conlleva naturalizarla y asumir una postura metafísica y omnicomprensiva. El enseñar este modelo como uno de carácter generalista o que el mismo representa una unidad que aplica a todos las áreas de intervención en trabajo social atenta contra el espíritu modernista de mantener una tensión entre la unidad y la diversidad (Adorno, 1989). Se estaría asumiendo una postura totalizante sobre el modelo de solución de problemas, posiblemente por que se desconoce la relación tensional entre lo único y lo diverso. El desarrollo del conocimiento científico en trabajo social es multi metodológico dada la complejidad del saber y sus diversas formas de conocerlo. Creer que la realidad se conoce con un método universal es no comprender la complejidad que es objeto de estudio. $^{8}$

\section{Teoria-práctica}

El énfasis del modelo de solución de problemas en lo instrumental, lo procesal y la empiria en el análisis de la política de Comunidades Especiales es cuestionable y entra en conflicto con otras maneras de construir conocimiento (epistemología) o interpretar un fenomenito

\footnotetext{
${ }^{8}$ Se utilizaron los siguientes 10 pasos de análisis: definir el problema, criterios para seleccionar alternativas, considerar soluciones, alternativas y adopción de soluciones, establecer tácticas y tareas, establecer plan de implantación, establecer sistemas de acción y recursos, evaluar si se resolvió problema, tomar decisiones si se resolvió o no el problema.
} 
social, en particular la propuesta por Popper (1985). Se respalda el supuesto popperiano de que un fenómeno social (práctica) puede ser comprendido si la explico desde una perspectiva conceptual, es decir si lo problematizo. La idea conceptual de Popper es parecida a la expuesta por Foucault (1989). Para Foucault, conocer un fenómeno conlleva comprender las reglas y criterios de que son las cosas y como estas funcionan. Al igual que Popper, Foucault cuestiona el pensamiento del sentido común (conjetura o lo concreto) como modo de conocer. Para ambos, el conocimiento auténtico es el conceptual.

La manera en que se plantearon los problemas de estudio en el documento se reproducía en la empiria lo mismo que estaba expuesto en la teoría y conceptos sobre la pobreza y la autogestión comunitaria. No había nada innovador en el análisis dado que los resultados reproducían lo mismo que estaba expuesto en la teoría. El modelo de solución de problemas utilizado para analizar destacó la importancia en recrear la experiencia, describir el proceso de legislación y no enfocó su estudio en la posibilidad de refutar los conceptos en torno a la pobreza y la autogestión comunitaria. Desde esta óptica, la estrategia debió ser identificar lo no explicado en la teoría para describir la práctica y así estudiar algo nuevo, aquí está lo innovador. Lo novedoso es producir lo que no se conoce o lo que no aplica a la realidad observada. Según Matus, la innovación auténtica parte de la tradición (1999). La tradición teórica no puede verse como un dado y sí como un hacer intelectual de romper con ella desde sus propias bases.

Es posible que un estudio epistemológico popperiano en torno al problema de la pobreza y la autogestión comunitaria hubiera llevado a otro tipo de resultado. En vez de representar indicadores de análisis que validarán lo ya establecido en la teoría, hubiera propuesto hipótesis de trabajo (derivado de la teoría) que contrastaran con la experiencia utilizando el proceso de refutación. El rol del trabajador social en este caso sería estudiar la experiencia en torno a los dos temas centrales de comunidades especiales (pobreza y autogestión) desde una perspectiva conceptual o problematizadora. Esto implica que el problema de la pobreza se iniciaría a partir una lógica conceptual y no de la mera experiencia o procesos instrumentales. 
El método epistemológico positivista popperiano es un avance frente a la dimensión epistemológica del racionalimo cientificista dado a que estudia el problema de la pobreza en una relación tensional con las teorías y conceptos que la avalan, mediada a través de la refutación. Tal proceder metodológico no es una división binaria, en la que la observación, la experiencia o la empiria está preponderantemente separada de la teoría y los conceptos. Además, tal metodología lleva a no limitar la práctica a una representación de las condiciones existentes de la pobreza a través de su magnitud o características. De igual manera, la práctica no sería aplicar el método de autogestión comunitaria como estrategia para resolver el problema de la pobreza en las Comunidades Especiales. Desde la perspectiva popperiana la práctica sería una reflexión conceptual de lo que no es la pobreza y la autogestión comunitaria a partir de los conceptos teóricos existentes.

Otra limitación del modelo de solución de problema es el énfasis en el análisis ${ }^{9}$ al estudiar la ley de Comunidades Especiales. La destreza intelectual de análisis parte de la idea que para comprender un fenómeno o una idea hay que descomponerla en fragmentos. Se comprende un fenómeno social cuando esta realidad la observo aisladamente. Así, que cuando se estaba analizando la legislación de Comunidades Especiales, el ejercicio que se estaba realizando era estudiar los diferentes fragmentos (en este caso pasos) con la esperanza de que tal procedimiento nos permitiera comprender la totalidad.

No obstante, un fenómeno social no necesariamente debe ser interpretado como un acto fragmentado y si relacional o por contradicciones. El concepto de falsación propuesto por Popper sugiere un criterio con visos dialécticos, dentro de la misma corriente epistemológica positivista, que permite interpretar determinado fenómeno social al confrontarlo con los conceptos teóricos. ${ }^{10}$ Para

\footnotetext{
${ }^{9}$ Análisis significa descomponer una totalidad en sus partes y estudiarlas por separado (Bloom, 1981).

${ }^{10}$ La refutación puede ser expuesta desde diversas corrientes epistemológicas. Según Adorno (1989), en la afirmación de algo se establece lo contrario de lo que se dice. En Popper (1985), se acepta la teoría como un dado. Conlleva la aplicación de un método hipotético-deductivo como un medio de contrastar críticamente las hipótesis o las teorías que explican lo que acontece en la experiencia.
} 
fines epistemológico, el método de la falsación resulta ser un instrumento crítico para analizar la política social de comunidades especiales debido a que nos aleja un poco de las corriente positivizante y cientificista sobre como construir conocimiento.

\section{Cuestionamiento conceptual y ético político}

En los dos temas anteriores se cuestionaron algunos supuestos epistemológicos de orientación positivistas del modelo de análisis de política pública utilizado en el estudio de la legislación de Comunidades Especiales. Se seleccionó la lógica de la refutación de Popper para apoyar su método de estudio en el análisis de la política pública. Claro está, esto no implica que estemos de acuerdo con sus supuestos conceptuales o creencia ético-política.

En esta sección nos enfocaremos en cuestionar algunos de los conceptos y supuestos ético-políticos del modelo de solución de problemas y sus implicaciones en el análisis de la política de Comunidades Especiales. Nos limitaremos al análisis crítico de las mismas sin pretender sugerir ofertas conceptuales o ético política concatenadas a lo epistemológico.

El método de análisis de solución de problema no asignó importancia en estudiar o desnaturalizar los conceptos de pobreza y autogestión comunitaria, como paso previo de estudio o de propuesta de intervención. El estudio fue instrumental y no conceptual. El análisis se centró en comprender como se manifestaba el problema de la pobreza sin interpretar sus significados ideológicos o supuestos epistemológicos. En el modelo racionalista de solución de problema no es pertinente estudiar lo ideológico o lo valorativo debido a que tales observaciones no tienen que ver con el método científico. Desde esta perspectiva la ciencia estudia los fenómenos sociales de manera objetiva y neutra, por consiguiente no tiene importancia científica los valores ideológicos que basamentan tal problema. Desde esta perspectiva, un analista de política pública no trabaja con valores y si con la realidad empírica que observa.

El problema de la pobreza se estudió utilizando los criterios del Censo de los Estados Unidos. Su énfasis fue describir las categorías de pobreza y sus manifestaciones. No se analizó el significado ideológico 
y conceptual de la pobreza y mucho menos los diversos enfoques epistemológicos que la explican. El análisis de la pobreza fue uno naturalizado o como una categoría pre establecida de lo que era la pobreza según el Censo de los Estados Unidos. Se consideró que entender la pobreza era conocer sus manifestaciones y no sus significados ocultos.

Al no estudiar los aspectos valorativos y conceptuales de la pobreza y la autogestión comunitaria, asumimos los postulados de la ciencia positivista de desconectar las raíces profundamente valorativas del trabajo social, profesión comprometida con la transformación social y con las clases oprimidas. Así, el método racionalista de solución de problemas para analizar la política pública de una legislación entra en contradicción con los valores que orientan la profesión de trabajo social.

Un segundo aspecto central en la legislación de Comunidades Especiales es el desarrollar en los miembros de la comunidad estrategias de apoderamiento para propiciar la autogestión comunitaria. Se hace evidente en la legislación que la estrategia se delimita al proceso educativo de organizar la comunidad. Nuevamente, la estrategia se enfoca en procedimientos y no en cuestionar el concepto de educar o educando (sujeto).

Educar no es enseñar formas de organizarse, no es aprender estrategias parlamentarias, no es aplicar técnicas para seleccionar entre alternativas y no es saber los procedimientos para solicitar servicios al Estado. En fin educar no es un saber meramente instrumental y sí una actitud auto conciente de reflexión. Educar es aprender a ser sujeto a través de la reflexión y la exposición pública de sus ideas. En este proceso no puede darse una relación de dependencia entre el organizador de comunidad y los miembros de la comunidad.

Desde este marco, es claro que el concepto de sujeto se convierte en eje central de todo esfuerzo de análisis de una política social, para enfrentar el problema de la pobreza en Puerto Rico. Tal proceso se dificulta si este proceso es dirigido por el Estado. La tendencia del Estado es hacia la auto reproducción y evitará todo esfuerzo por la independencia intelectual de los miembros de la comunidad por 
develar críticamente las contradicciones de la pobreza y sus posibles contingencias.

Se requiere de un proceso formativo en la cual el sujeto, como concepto, sea eje central de cuestionamiento de su situación y de su entorno. La oferta fenomenológica, a través del pensamiento de Husserl (1973, p 234), es una opción para retomar el rol del sujeto en las Comunidades Especiales. La fenomenología Husseriana fomenta el desnaturalizar el entorno a través de una reflexión que lleve al individuo a tener una imagen de sí mismo en contraste con su entorno social. Es la lucha interna de quien soy con relación a mis circunstancias. En la medida que puedo conceptuar mi yo (¿Quién soy y cuáles son mis posibilidades de ser lo que quiero ser?) en relación con mi entorno es que lo natural y lo categorial se desnaturaliza y se re categoriza.

La autoconciencia lleva al sujeto a un compromiso ético de responsabilizarse por sus ideas y acciones. Tal compromiso ético es consistente con el concepto de apoderamiento y de autogestión. Independientemente que la contingencia de los sujetos sea limitada. El sujeto tiene opciones para enfrentarlas y transformarlas. No se justifica el inmovilismo ético, debido a que la autoconciencia permite la transformación de mundo, independientemente lo reducido o ampliado de las posibles contingencias. A mayor autoconciencia, mayor posibilidad de abrir posibilidades de cambio del entorno.

El compromiso conceptual y ético de la legislación de Comunidades Especiales y de la profesión de trabajo social es concebir a los miembros de las Comunidades Especiales como sujeto. El limitar la educación comunitaria a comprensión de normas, procedimientos, reglas es restar posibilidades al desarrollo de sujetos en las comunidades. De lo contrario, la estrategia de intervención comunitaria puede contener una retórica modernista y sus resultados ser una nueva metafísica totalizante, en la cual los procedimientos se convierten en una unidad en sí misma sin opciones en la variedad.

\section{Problema de estudio}

En esta sección se derivan las conclusiones más destacadas del argumento con el propósito de justificar el problema de análisis de la 
política pública de Comunidades Especiales. A partir de la argumentación de este escrito se reconstruyen los posibles problemas que podrían ser objeto de análisis de la política pública de Comunidades Especiales. Nos concentraremos en las conclusiones centrales del estudio y no en las discusiones secundarias.

El modelo de solución de problema utilizado para analizar y evaluar la legislación de comunidades especiales no es un método de estudio adecuado para comprender la complejidad de la pobreza en Puerto Rico. El concentrar sus esfuerzos en lo instrumental y no considerar lo conseptual, se limita a resaltar lo procesal sin entender la sustantividad de lo que es objeto de estudio. La intervención que se deriva de este proceso instrumental tiene pocas probabilidades de producir resultados debido a su falta de fundamentación y pertinencia. El utilizar el modelo de solución de problema como método único de análisis de la política pública puede llevar a actitudes totalizantes que no son cónsonas con el mismo pensamiento positivista. Las presentaciones anteriores a esta son dos buenos ejemplos de cómo analizar un fenómeno social, trascendiendo lo instrumental y desnaturalizando la complejidad de la pobreza, proceso intelectual básico para formular una política pública.

El método de refutación propuesto por Karl Popper es una estrategia adecuada, entre otras, para estudiar el problema de la pobreza a partir una lógica conceptual y no de la mera experiencia o procesos instrumentales. El rol del trabajador social en este caso sería estudiar la experiencia en torno a los dos temas centrales de comunidades especiales (pobreza y autogestión) desde una perspectiva conceptual o problematizadora. Por consiguiente, se propone las siguientes preguntas de estudios para orientar el análisis de la política pública de Comunidades Especiales:

- ¿Cuál es el significado de la pobreza en puerto rico desde una perspectiva analítica no instrumental y sí por negatividad?

- ¿Cuáles son los conceptos que se contraponen en la legislación de comunidades especiales que posibilitan o limitan la supresión de la pobreza en Puerto Rico? 
- ¿La pobreza en Puerto Rico es un problema o una contradicción?

- ¿Cuáles estrategias educativas no deben utilizarse en las Comunidades Especiales para formar líderes comunitarios basados en el concepto de sujeto?

Estas preguntas de estudios no tienen contestaciones al momento y posiblemente las contestaciones a estas o algunas de ellas propicien nuevas interrogantes sin que ello implique una contestación final. No obstante, lo relevante de estas interrogantes es que las mismas son el resultado de un análisis conceptual y no empírico. De igual manera, sus contestaciones nos permitirán ver con mayor claridad, en determinado momento histórico, el significado de lo que es la pobreza como fenómeno social y el tipo de intervención requerido para lidiar con tal fenómeno. No hay una llegada final y si una construcción constante sin saber su llegada final.

\section{REFERENCIAS}

Adorno, T. (1989). Tres estudios sobre Hegel. Editorial Taurus. Madrid. Pág.15-75.

Bardack, E. (2000). A practical guide for policy analysis. New York: Chatham House Publishers.

Bloom, B. (1981). Taxonomía de los objetivos educativos: La clasificación de las metas educacionales. ( $8^{\mathrm{a}}$. Ed.) Argentina: El Ateneo Editorial.

Durkheim, E. (1978). Las reglas del método sociológico. Buenos Aires: Edic. La Pléyade, pág. 12.

Estremera, R. (2002). Análisis y Recomendaciones de Política Pública en torno a la Ley de Comunidades Especiales como estrategia para resolver el problema de la pobreza en Puerto Rico. Escrito sometido como requisito del curso TSOC 8001. 
Foucault, M. (1989). Vigilar y Castigar. Barcelona: Editorial La Piqueta. P. 11-36.

Gilbert, F.H. Specht. (1984). Dimensions of Social Welfare. (1974). Englewood Cliffs: New Jersey.

Guardiola, D. y Serra, José A. (2002). Política social y trabajo social en Puerto Rico: Desafíos y alternativas para siglo XXI. Puerto Rico: Publicaciones Puertorriqueñas

Guardiola, D. (1998). Trabajo Social en Puerto Rico: ¿Asistencia, desarrollo, transformación? Puerto Rico: Editorial Edil.

Husserl, E. (1973). La filosofía como ciencia estricta: La filosofía como autorreflexión de la humanidad. Buenos Aires: Editorial Nova. págs.126-134.

Jones, C. (1984). An introduction to the study of public policy. Nonterrey, CA: Brooks/Cole. Ch. 1-2. p. 5-37

Ley \# 1 Estado Libre Asociado de Puerto Rico. (2001). Ley para el Desarrollo Integral de las Comunidades Especiales de Puerto Rico. Ley 1 de marzo de 2001.

Matus, T. (1999). Propuestas contemporáneas en trabajo social: Hacia una intervención polifónica. Buenos Aires: Editorial Espacio. Introducción y Cap. Primero.

Matus, T. (1994). Cuando la epistemología no basta. Revista Acto Social. No. 4 Córdova.

Popper, K. (1985). La lógica de la investigación científica. Madrid: Editorial Tecnos. 
